\title{
Remote Power Control of Wireless Network Interfaces
}

\author{
Andrea Acquaviva ${ }^{1}$, Tajana Simunic ${ }^{2}$, Vinay Deolalikar ${ }^{2}$, and Sumit Roy ${ }^{2}$ \\ 1 Istituto di Scienze e Tecnologie dell'Informazione \\ Universita' di Urbino, Italia \\ acquaviva@sti.uniurb.it, \\ 2 Hewlett-Packard Laboratories \\ 1501 Page Mill Road \\ Palo Alto, CA 94304, USA \\ \{tajana.simunic, vinay.deolalikar, sumit.roy\}@hp.com
}

\begin{abstract}
This paper presents a new power management technique aimed at increasing the energy efficiency of client-server multimedia applications running on wireless portable devices. We focus on reducing the energy consumption of the wireless network interface of the client by allowing the remote server to control the power configuration of the network card depending on the workload. In particular, we exploit server knowledge of the workload to perform an energy-efficient traffic reshaping, without compromising on the quality of service. We tested our methodology on the SmartBadge IV wearable device running an MPEG4 streaming video application. Using our technique we measured energy savings of more than $67 \%$ compared to no power management being used on the WLAN interface. In addition, we save as much as $50 \%$ of energy with respect to the standard $802.11 \mathrm{~b}$ power management. All of the energy savings are obtained with no performance loss on the video playback.
\end{abstract}

\section{Introduction}

Portable devices spend a considerable amount of energy in order to support power hungry peripherals e.g. wireless local area network (WLAN) interfaces, and liquid crystal displays (LCD). A good example of such a device is the SmartBadge IV [21] wearable computer. It consists of a StrongARM-1110 processor and SA-1111 coprocessor, memory, WLAN interface, audio codec and 2.2" LCD. Depending on the network traffic, the WLAN accounts for as much as $63 \%$ of the overall system power consumption.

One way to reduce the energy consumption is to use the power management included in the $802.11 \mathrm{~b}$ standard (802.11b PM) [4]. In the standard, an access point (AP) transmits a beacon every $100 \mathrm{~ms}$, followed by a traffic indication map (TIM). Each client checks the TIM for its turn to send or receive data. When not communicating, the WLAN goes into the doze mode until the next beacon. Unfortunately, the protocol power management is not very effective. First, the 
energy efficiency of the 802.11b PM decreases and receiver wait times increase with more mobile hosts, since multiple concurrent attempts at synchronization with the beacon cause media access contention. Second, the response time of the wireless link with $802.11 \mathrm{~b}$ PM grows because of the delay imposed by sleep periods [22]. These two issues can be resolved by careful scheduling of communication between the server and the client WLAN. Lastly, in a typical wireless network, broadcast traffic can significantly reduce the chances to enter the doze mode. Figure 1 shows the power consumption of a WLAN card with $802.11 \mathrm{~b}$ PM enabled under light and heavy network broadcast traffic conditions. Clearly, as the amount of broadcast traffic increases, the WLAN spends a large amount of energy listening to it, even if no other application is running on the device. As a result, very little or no energy savings are obtained. One way to solve this problem is to turn off the card. It is important to schedule data transmission carefully, since the overhead of waking up the WLAN from the off state is large.

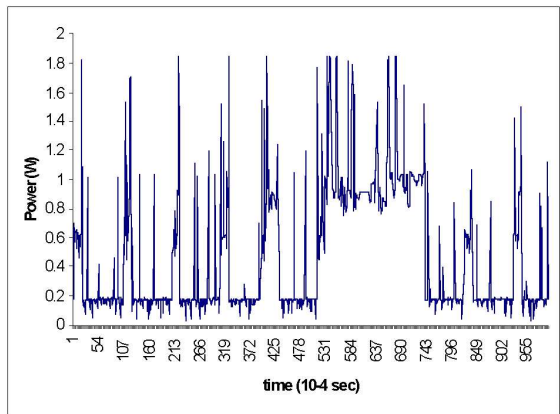

a) light traffic

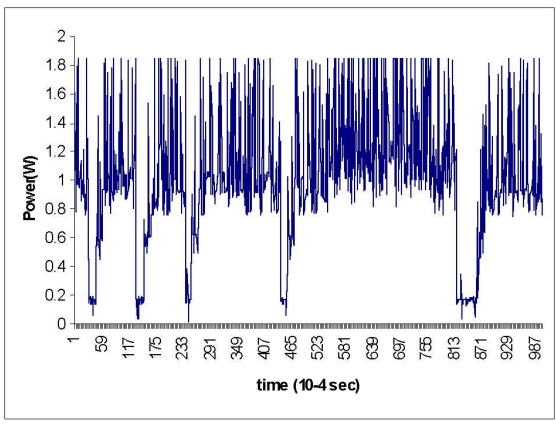

b) heavy traffic

Fig. 1. 802.11 PM under different broadcast traffic conditions

Many current wireless local area networks are organized in a client-server fashion. Multiple WLAN clients connect to wired servers via APs. Servers are great candidates for efficient scheduling of data transmission to clients as they are not power constrained, and know both wired and wireless network conditions.

In this work, we present a server controlled power management strategy. Our technique exploits server knowledge of the workload, traffic conditions and feedback information from the client in order to minimize WLAN power consumption. Our methodology is applicable to a wide variety of applications, ranging from video and audio streaming, to web browsing and e-mail. We define two new entities: a server power manager (server PM) and a client power manager (client PM). Server PM uses the information obtained from the client and the network to control the parameters of $802.11 \mathrm{~b}$ PM and to perform energy efficient traffic reshaping so that WLAN can be turned off. Client PM communicates through a dedicated low-bandwidth link with the server PM and implements power con- 
trols by interfacing with device drivers. It also provides a set of APIs that client programs can use to provide extra information to the server.

In order to illustrate the effectiveness of our approach, we tested our methodology with a streaming video application. By using our approach with this application, we can exploit the server knowledge of stream characteristics. We show that when our methodology is implemented on both the server end, and on the client end, we measure savings of more than $67 \%$ in power with respect to leaving the card always on, and more than $50 \%$ relative to using default $802.11 \mathrm{~b}$ PM. Even larger savings are possible for applications that inherently have longer idle periods, such as e-mail or web browsing. Our methodology can also be easily extended to manage other system components (e.g. CPU).

The rest of the paper is organized as follows. Section 2 presents some related work. Section 3 gives an overview of the proposed methodology and describes server and client power managers. Section 4 presents experimental results and Section 5 concludes the paper.

\section{Related Work}

The wireless network power optimization problem has been addressed at different abstraction layers, starting from physical, to system and application level. Energy efficient channel coding and traffic shaping to exploit battery lifetime of portable devices were proposed in [3]. A physical layer aware scheduling algorithm aimed at efficient management of sleep modes in sensor network nodes is illustrated in [17]. Energy efficiency can be improved at the data link layer by performing adaptive packet length and error control [8]. At the protocol level, there have been attempts to improve the efficiency of the standard $802.11 \mathrm{~b}$, and proposals for new protocols $[5,6,19]$. Packet scheduling strategies can also be used to reduce the energy consumption of transmit power. In [14] authors propose the $E^{2} W F Q$ scheduling policies based on Dynamic Modulation Scaling. A small price in packet latency is traded for the reduced energy consumption.

Traditional system-level power management techniques are divided into those aimed at shutting down components and policies that dynamically scale down processing voltage and frequency $[20,1]$. Energy-performance trade-offs based on application needs have been recently addressed [7]. Several authors exploit the energy-QoS trade-off $[12,22,11]$. A different approach is to perform transcoding and traffic smoothing at the server side by exploiting estimation of energy budget at the clients [16]. A new communication system, consisting of a server, clients and proxies, that reduces the energy consumption of $802.11 \mathrm{~b}$ compliant portable devices by exploiting a secondary low-power channel is presented in [18]. Since multimedia applications are often most demanding of system resources, a few researchers studied the cooperation between such applications and the OS to save energy $[9,2,15,10]$.

We present a new methodology, where server knowledge of the workload is exploited to control the power configuration of the radio interface. Compared to physical and protocol layer strategies, the power control is performed at the ap- 
plication level, so it does not require hardware modifications. Compared to clientcentric approaches, we exploit additional information available at the server, and thus obtain large energy savings without loosing performance. Moreover, with respect to previous application-driven policies, our infrastructure can be used with a wide range of applications, since it exploits very common parameters.

\section{Server Controlled Power Management}

Our methodology exploits server knowledge of the workload, traffic conditions and feedback information from the client in order to minimize power consumption. The server schedules communication sessions with the client based on the knowledge of both, the wireless and wired networks, e.g favorable channel conditions or channel bandwidth capabilities. When broadcast traffic needs to be monitored, the server can enable the 802.11 PM. Alternatively, it can coordinate the shut down of WLAN and perform on-time wake-up, thus avoiding the performance penalty typically incurred by client-centric approaches. Our application driven infrastructure can be also be used to manage power consumption in stand-alone and ad-hoc applications. The power control strategy can easily be extended to include other system components, such as peripherals or the CPU.

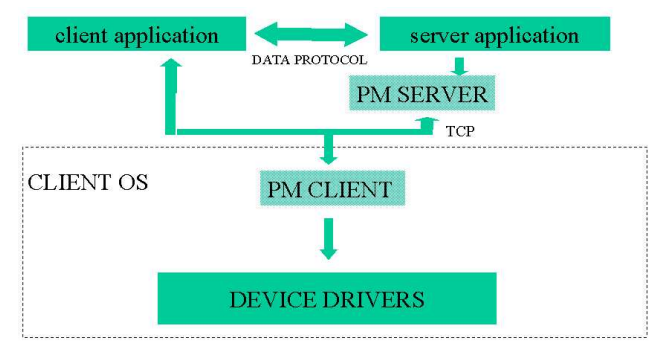

Fig. 2. Server Controlled PM Architecture

In order to exploit the extra information available at the server and the client, the traditional client-centric power manager model has to be extended. Two different power managers are defined: one running on the client (Client PM) and the other on the server (Server PM). The two PMs exchange power control information through a dedicated TCP connection. As shown in Figure 2, the client PM interfaces directly with the drivers on the portable device. It also collects client application dependent information. The server PM interfaces with the server application and the client PM.

Figure 3 shows the communication protocol between the server and client. Control commands are issued by the server PM, interpreted by the client PM, and translated into appropriate device driver calls. Upon request from the server PM, device specific information is fetched by the client PM. Application specific information can also be retrieved by the client PM via API calls. 


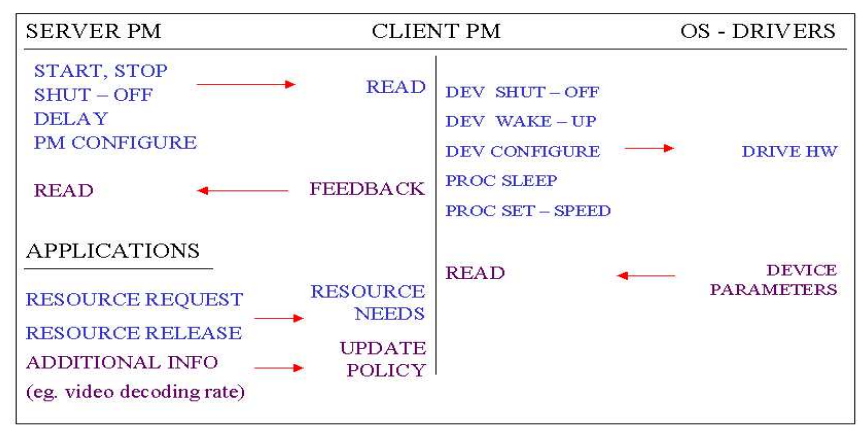

Fig. 3. Communication protocol

\subsection{Server Power Manager}

The server PM interfaces with the application in order to exploit information available on the host system, in addition to the knowledge of the overall network conditions and the specific feedback information provided by each client. Based on all this knowledge, the server PM controls the power configuration of the WLAN card by communicating with the client PM. The power control commands used include: i) switch-off the WLAN; ii) set the off time; iii) enable the $802.11 \mathrm{~b}$ PM policy; iv) set $802.11 \mathrm{~b}$ PM parameters, e.g. the period between wake ups and the timeout before going back to doze mode. The server PM enables:

Client Adaptation. After the application server initializes the server PM, an additional set of APIs becomes available. The initialization routine places the server PM into a state of waiting for incoming client PM requests. Each accessing client PM has a dedicated TCP connection to the server PM. The client PM informs the server PM about the application e.g. input buffer size, the expected value and variance of the service rate, as well as network interface specific information e.g. the WLAN card status and its on/off transition time.

Traffic Adaptation. Server PM monitors both the wired and wireless traffic conditions with minimum overhead. By accounting for the broadcast packet rate, the server decides when to enable the $802.11 \mathrm{~b}$ PM. For example, in very light traffic conditions, the $802.11 \mathrm{~b}$ PM might be used instead of a switch-off policy.

Traffic Shaping. The server PM schedules transmissions to the client in bursts, in order to compensate for the client performance and energy overheads during transitions between on and off states. The client WLAN card is switched off once the server has sent a burst of data designed to keep the application busy till the next communication burst. The burst size and delay between bursts are precomputed at the server. The delay should be large enough to almost empty the client input buffer, while the burst size should avoid overflow while keeping the buffer sufficiently filled. This maximizes the off time of the card and reduces the number of transitions between on and off states. The time for the buffer to empty, $D_{\text {burst }}$ is the ratio of the total number of packets in the burst, $S I Z E_{\text {burst }}$, 
to the average service rate (or buffer depletion rate) at the client, $\lambda_{s, \text { mean: }}$

$$
D_{\text {burst }}=\frac{S I Z E_{\text {burst }}}{\lambda_{s, \text { mean }}}
$$

The total delay between bursts, $D_{\text {burst }}^{\mid}$is:

$$
D_{\text {burst }}^{\mid}=D_{\text {burst }}-T_{\text {tran }}-T_{\text {cushion }}
$$

Where $T_{\text {tran }}$ is the time needed for the transitions between WLAN on and off states, $T_{\text {cushion }}$ is the "cushion" time so that the buffer does not ever empty completely. The cushion time helps accommodate variations of the service rate and arrival rate, $10 \%$ was found to be sufficient for most test cases. The total energy saved if the client WLAN is turned off is:

$$
E_{\text {tot }}=P_{o n} \cdot T_{o n}+P_{o f f} \cdot T_{o f f}+P_{t r a n} \cdot T_{t r a n}
$$

Here $P_{t r a n}$ is the transition power (hardware dependent). $T_{\text {tran }}$ can be computed by multiplying the duration of one transition to the active state, $T_{\text {wake-up }}$, by the total number of transitions, $N_{\text {tran }}$. The number of transitions depends on the size of the burst and the total size of streamed file.

If $\lambda_{a, \text { mean }}$ is the average arrival rate at the client input buffer, the total time to send a burst of data to the client will be:

$$
T_{\text {on }}=\frac{S I Z E_{\text {burst }}}{\lambda_{a, \text { mean }}}
$$

Finally, we can obtain the total energy consumed with our methodology:

$$
E_{\text {tot }}=P_{\text {on }} \cdot \frac{S I Z E_{\text {burst }}}{\lambda_{a, \text { mean }}}+P_{\text {off }} \cdot \frac{S I Z E_{\text {transfer }}}{\lambda_{s, \text { mean }}}+P_{\text {tran }} \cdot T_{\text {tran }}
$$

In addition to scheduling communication so that the client WLAN can be turned off with no performance overhead, we can also perform the same scheduling while $802.11 \mathrm{~b}$ PM is enabled. This allows us to save energy during the burst periods, but the overall burst time grows because of the decreased responsiveness and increased contention probability between data and broadcast packets.

\subsection{Client Power Manager}

The client power manager communicates with the server PM, and also interfaces with the device drivers and client applications. The main client PM tasks are:

Server Interface The client application decides when to set-up the communication between the server PM and the client PM. Once established, the client application provides the information to be forwarded to the server e.g. the buffer size and the depletion rate. The device drivers report the main characteristics 
of the devices to be managed, e.g. the transition time between on and off states for client WLAN.

Device Interface The client PM calls the appropriate device driver function depending on the command sent by the server PM. Possible actions taken by the client include changing the parameter of the $802.11 \mathrm{~b}$ PM, switching the WLAN on and off, and reading the interface statistics such as the signal to noise ratio. The client PM can also interact with the CPU by changing its power mode or setting its clock speed.

Application Interface Applications can feedback information that can be exploited by both the server and the client PM. Examples include sending the current backlog level to the server, so that thee server knows exactly how much data to provide in a burst in order to refill the buffer; or providing the buffer size. The application could directly request a WLAN wake-up when its input buffer reaches a minimum value.

Application Driven Infrastructure The client PM can also be used in stand-alone mode (with no server). While in this mode, the applications provide their resource needs to the power manager. The PM then turns on or off devices appropriately. Some of the overhead needed to turn on a device can be masked, as many applications have extra latency due to the initial set-up.

\section{Experimental Results}

The streaming media server used for this work is a research prototype developed at Hewlett-Packard Laboratories. Real Time Streaming Protocol (RTSP) is used for session initiation and termination between the client and server. The media data units are carried using Real-time Transport Protocol (RTP) over User Datagram Protocol (UDP). Timestamps of the individual video frames are used to determine the deadline for sending data packets sent from the server to the client. The server can exploit client buffering capabilities to reschedule packet transmission times based on a cost function. In our experiments, the server transmits MPEG4 video data to a portable client device via WLAN.

We use two different benchmarks in our tests. Benchmark 1 has 12 bursts and runs at 15 frames/sec, while Benchmark 2 has 402 bursts at 30 frames/sec. The first benchmark has a large number of single packet frames, while the second benchmark has many large multi-packet frames. The delay between bursts is:

$$
D_{\text {burst }}=\text { frame_time } \cdot\left(\frac{n_{1}}{1}+\frac{n_{2}}{2}+\frac{n_{3}}{3}+\ldots+\frac{n_{M}}{M}\right)
$$

Here frame_time is the interval between frames, $n_{i}$ is the number of frames consisting of $i$ packets and $M$ is the maximum number of packet per frame. Figure 4 shows that the Benchmark 1 has a delay of around 4 seconds, while the Benchmark 2 has a shorter delay because the frames are more complex. Based on the delay computation, the server transmits a burst of data to the client followed by the command to switch off WLAN and the length of time until the next transmission. The client responds by turning off the WLAN and turning it 




a) Benchmark 1

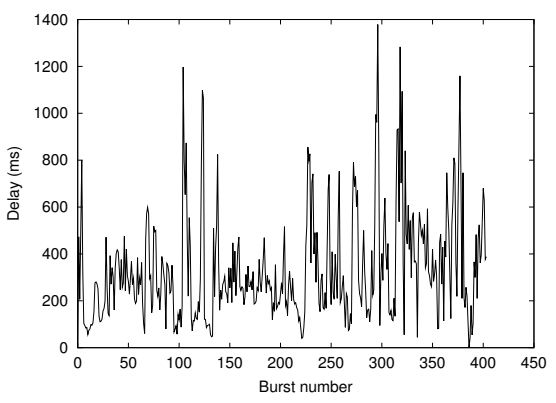

b) Benchmark 2

Fig. 4. Computed burst delays for each benchmark

back on at the pre-scheduled time. As a result, no performance penalty due to longer turn on time is incurred.

The approach was tested by measuring power consumption of a SmartBadge IV wearable device, equipped with a CISCO Aironet 350 PCMCIA WLAN card. The off/on transition time was measured at $300 \mathrm{~ms}$, with an average transition power consumption of $0.1 \mathrm{~W}$. The server is connected directly to an AP, and the network is completely isolated in order to perform repeatable experiments. Broadcast traffic is introduced in a controlled manner by using real traces collected from other open networks. Power measurements are performed using a DAQ board accumulating and averaging current and voltage samples (10 ksamples/sec). All measurements include the overhead imposed by our power management protocol.

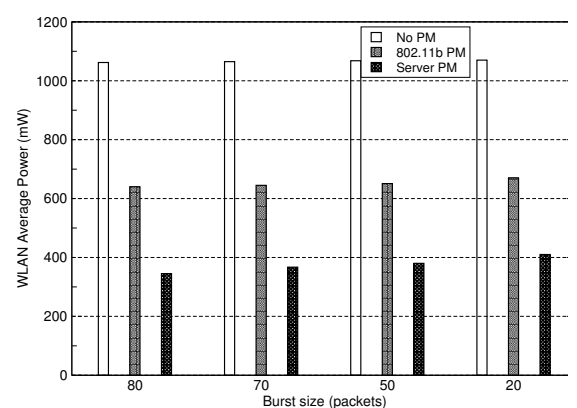

a) Benchmark1

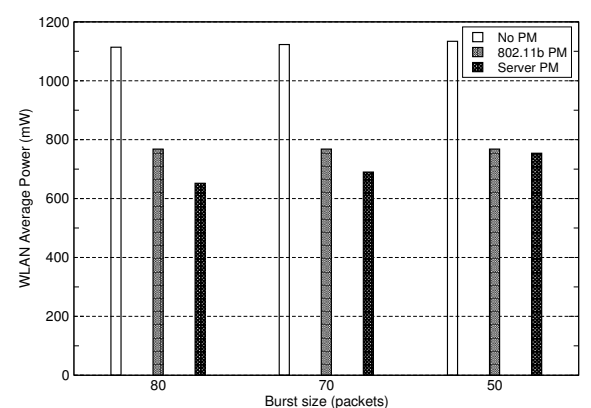

b) Benchmark2

Fig. 5. Power consumption for each benchmark

First we computed the average power consumed by the WLAN card when receiving the video stream with different burst sizes for medium broadcast traffic conditions. The experiment is performed for multiple situations, WLAN with no PM, only 802.11b PM, and with server PM. From Figure 4, for Benchmark 1, the 
server controlled approach saves $67 \%$ of average power compared to no PM, and $50 \%$ compared to the default $802.11 \mathrm{~b}$ PM. The average power savings increase as the burst size increases, since this enables longer times between bursts, thus better compensation for the transition delay between the WLAN on and off states. In all three cases, the video plays back in real time, thus the reported average power savings directly correspond to energy savings. For Benchmark 2, the delays are very short, however, we still save $41 \%$ of power with respect to leaving the card always on and $15 \%$ with respect to the standard $802.11 \mathrm{~b}$ power management protocol with bursts of 80 packets. We cannot use a burst size smaller than 50 packets, since the computed delays are too short and the card can never be switched off.



Fig. 6. Power consumption with different traffic levels

Figure 6 presents the average power consumed during streaming video under different broadcast traffic conditions. In the plot we show the difference between using only the default $802.11 \mathrm{~b}$ PM and server controlled switch off policy for the WLAN card (with no 802.11b PM). The switch off policy is almost insensitive to the traffic conditions, while the $802.11 \mathrm{~b}$ PM performs better only in light traffic conditions. Clearly, our server controlled power management approach is more efficient than either policy alone as it always selects the best of the two policies.

\section{Conclusion}

In this work we presented a new methodology to improve the energy efficiency of portable wireless systems that operate in a client-server fashion. Our system enables the server to exploit knowledge of the workload, traffic conditions and feedback information from the client in order to minimize client WLAN power consumption. We tested our methodology on the SmartBadge IV wearable device running a streaming video application. Our server controlled approach saves $67 \%$ of energy as compared to leaving the client WLAN card always on, and $50 \%$ as compared to the simple 802.11 b PM policy. 


\section{References}

1. A. Acquaviva, L. Benini, B. Riccó, "Software Controlled Processor Speed Setting for Low-Power Streaming Multimedia," IEEE Trans. on CAD, Nov. 2001.

2. F. Bellosa, "Endurix: OS-Direct Throttling of Processor Activity for Dynamic Power Management," Technical Report TR-I4-99-03, University of Erlangen, June 1999.

3. C. Chiasserini, P. Nuggehalli, V. Srinivasan, "Energy-Efficient Communication Protocols", Proc. of DAC, 2002.

4. IEEE LAN/MAN Standards Committee, "Part 11: Wireless LAN MAC and PHY Specifications: Higher-Speed Physical Layer Extension in the 2.4 GHz Band", 1999.

5. C. Jones, K. Sivalingam, P. Agrawal, J. Chen, "A Survey of Energy Efficient Network Protocols for Wireless Networks", Proc. of DATE, 1999.

6. R. Krashinsky, H. Balakrishnan, "Minimizing Energy for Wireless Web Access with Bounded Slowdown", Proc. of MOBICOM, 2002.

7. R. Kravets, P. Krishnan, "Application-Driven Power Management for Mobile Communication," Proc. of WINET, 1998.

8. P. Lettieri, C. Schurgers, M. Srivastava, "Adaptive Link Layer Strategies for Energy Efficient Wireless Networking", Wireless Networks, no. 5, 1999.

9. J. Lorch, A. J. Smith, "Software Strategies for Portable Computer Energy Management," IEEE Personal Communications, June 1998.

10. Y. Lu, L. Benini, G. De Micheli, "Operating System Directed Power Reduction," Proc. of ISLPED, July 2000.

11. C. Luna, Y. Eisenberg, R. Berry, T. Pappas, A. Katsaggelos, "Transmission Energy Minimization in Wireless Video Streaming Applications," Proc. of Asilomar Conf. on Signals, Systems, and Computers, Nov. 2001.

12. R. Min, A. Chandrakasan, "A Framework for Energy-Scalable Communication in High-Density Wireless Networks," Proc. of ISLPED, 2002.

13. T. Pering, T. Burd, R. Brodersen, "Voltage Scheduling in the lpARM Microprocessor System", Proc. of ISLPED, July 2000.

14. V. Raghunathan, S. Ganeriwal, C. Schurgers, M. Srivastava, " $E^{2} W F Q$ : An Energy Efficient Fair Scheduling Policy for Wireless Systems", Proc. of ISLPED, 2002.

15. J. Flinn, M. Satyanarayanan, "Energy-aware adaptation for mobile applications," Proc. of SOSP, Dec. 1999.

16. P. Shenoy, P. Radkov, "Proxy-Assisted Power-Friendly Streaming to Mobile Devices," Proc. of $M M N C$, Jan. 2003.

17. E. Shih, P. Bahl, M. Sinclair, "Dynamic Power Management for non-stationary service requests", Proc. of MOBICOM, 2002.

18. E. Shih, S. Cho, N. Ickes, R. Min, A. Sinha, A. Wang, A. Chandrakasan, "Physical Layer Driven Protocol and Algorithm Design for Energy Efficient Wireless Sensor Networks", Proc. of SIGMOBILE, 2001.

19. K. Sivalingam, J. Chen, P. Agrawal, M. Srivastava, "Design and Analysis of lowpower access protocols for wireless and mobile ATM networks", Wireless Networks, no. 6,2000 .

20. T. Simunic, L. Benini, P. Glynn, G. De Micheli, "Event-driven Power Management," IEEE Trans. on CAD, July 2001.

21. M. T. Smith and G. Q. Maguire Jr., "SmartBadge/BadgePad version 4", HP Labs and Royal Institute of Technology (KTH), http://www.it.kth.se/ maguire/badge4.html, date of access: 2003-06-11.

22. E. Takahashi, "Application Aware Scheduling for Power Management on IEEE 802.11" Proc. of Intl. Performance, Computers, and Communications Conf., Feb. 2000. 